\title{
Philonsorbonne
}

13 | 2019

Année 2018-2019

\section{La représentation comme fiction chez Jeremy Bentham}

\section{Ludmilla LORRAIN}

\section{(2) OpenEdition}

1 Journals

Édition électronique

URL : https://journals.openedition.org/philonsorbonne/1171

DOI : 10.4000/philonsorbonne. 1171

ISSN : 2270-7336

Éditeur

Publications de la Sorbonne

Édition imprimée

Date de publication : 30 janvier 2019

Pagination : 75-94

ISSN : 1255-183X

\section{Référence électronique}

Ludmilla LORRAIN, «La représentation comme fiction chez Jeremy Bentham », Philonsorbonne [En ligne], 13 | 2019, mis en ligne le 06 février 2019, consulté le 11 juin 2021. URL : http://

journals.openedition.org/philonsorbonne/1171; DOI : https://doi.org/10.4000/philonsorbonne. 1171

(c) Tous droits réservés 


\title{
La représentation comme fiction chez Jeremy Bentham
}

\author{
Ludmilla LORRAIN
}

\section{Introduction}

Le fait que le philosophe Jeremy Bentham considère la «démocratie pure représentative » comme la meilleure forme de gouvernement peut sembler tout à fait banal. Il est en effet usuel d'associer le régime démocratique à la représentation politique - celui-ci ayant besoin de ce mécanisme pour se traduire institutionnellement. Pourtant, au tournant $\mathrm{du} \mathrm{XIX}^{\mathrm{e}}$ siècle, et alors que le régime démocratique reste largement considéré comme une forme politique à éviter ${ }^{1}$, cette affirmation possède une radicalité certaine.

Chez Bentham, cette supériorité de la démocratie sur les autres formes de gouvernement tient en premier lieu à l'adoption du principe d'utilité. Car le plus grand bonheur du plus grand nombre ne peut se traduire politiquement qu'au sein d'un régime mettant en œuvre l'impartialité exigée par ce principe - stipulant que «chacun doit compter pour un, et aucun pour plus d'un ${ }^{2} »$. À cet égard, et contrairement aux fondateurs des régimes représentatifs - qui voyaient dans le recours à la représentation politique

\footnotetext{
1. L'ouvrage Re-imagining Democracy in The Age of Revolutions, America, France, Britain, Ireland 1750-1850 (sous la direction de J. Innes et M. Philp, Oxford, Oxford University Press, 2013), qui porte sur les usages de la notion de démocratie de la fin du XVIII ${ }^{\mathrm{e}}$ siècle au premier XIX ${ }^{\mathrm{e}}$, met tout à fait en évidence la manière dont, au moins jusqu'aux années 1820, la démocratie ne fait pas partie de l'horizon politique - le qualificatif «démocrate » étant d'ailleurs utilisé presque exclusivement par les adversaires de la réforme dans le but de discréditer leurs adversaires politiques.

2. Bentham, Rationale of Judicial Evidence, in Works of Jeremy Bentham, J. Bowring (dir.), Edinburgh, William Tait, 1843, vol. VII, p. 334.
} 
un moyen de soustraire à l'influence du peuple le pouvoir souverain -, la démocratie représentative n'est pour lui ni un substitut à la démocratie directe, ni une forme politique supérieure, qui opérerait une discrimination des meilleurs, tout en disqualifiant un peuple dont les jugements seraient, par définition, empreints d'irrationalité ${ }^{3}$. Si c'est le meilleur régime, c'est parce que son fondement est démocratique. La démocratie représentative proposée par Bentham constitue ainsi l'une des premières propositions articulées visant à faire des régimes représentatifs des démocraties.

Ce choix le conduit donc naturellement à réfléchir à la manière de mettre en œuvre la représentation dans un contexte démocratique. Semblant à cet égard tenir compte des analyses de J.-J. Rousseau au chapitre XV du livre III du Contrat Social, Bentham souligne que les ambivalences du mécanisme représentatif permettent le plus souvent aux gouvernants d'usurper le pouvoir souverain, et de sacrifier l'intérêt du plus grand nombre au profit de leur intérêt. C'est pourquoi il est essentiel de déterminer le plus clairement possible les modalités d'implantation de ce mécanisme.

En effet, et comme l'ont notamment souligné les travaux de H. Pitkin ou de P. Rosanvallon ${ }^{4}$, le concept de représentation, loin d'être univoque, peut désigner des réalités extrêmement différentes. Aussi la mise en œuvre d'institutions représentatives dans un contexte démocratique confronte-t-elle à deux types de questionnements. Premièrement, si «représenter» signifie « rendre présent l'absent», il faut alors s'interroger sur ce qui, de l'absent, doit être représenté. S'agit-il ainsi de produire un reflet fidèle des opinions existant dans une communauté donnée, ou bien d'affirmer que seuls les représentants peuvent formuler un intérêt commun qui ne saurait préexister à cette mise en représentation? Par ailleurs, la question du contenu de l'activité de représentation doit être décidée, en interrogeant particulièrement le lien qui unit les électeurs à leur représentant. Car l'on peut aussi bien attendre de lui qu'il substitue sa volonté à celle du représenté, défendant ses intérêts en fonction de la perception qu'il s'en fait, ou bien plutôt qu'il porte ailleurs une volonté déjà formulée ${ }^{5}$. Ces questions sont essentielles, car la manière d'y répondre affecte directement la dimension démocratique des régimes considérés.

3. On peut rappeler les formules de Madison dans le Fédéraliste, 10, où l'on voit que ce qui fait l'infinie supériorité du régime représentatif est qu'il permet « d'affiner et d'élargir l'esprit public en le faisant passer par la médiation d'un corps choisi de citoyens dont la sagesse peut discerner les vrais intérêts de leur pays » et qu'ainsi «il est bien possible que la voix publique, articulée par les représentants du peuple, puisse être plus accordée au bien public que si elle était prononcée par le peuple lui-même assemblé dans ce but ». Nous trouverions des remarques du même ordre dans de nombreux écrits de Sieyès, voir par exemple Dire de l'abbé Sieyès sur la question du véto royal.

4. H. Pitkin, The Concept Of Representation, Berkeley, Los Angeles, London, University of California Press, 1967 ; P. Rosanvallon, Le Peuple introuvable. Histoire de la représentation démocratique en France, Paris, Gallimard, 1998.

5. Sur ces questions, le travail de H. Pitkin, au chapitre 6 de son ouvrage The Concept of Representation, qui s'attache à dégager les différents sens de la représentation à partir d'une étude des figures du messager, de l'avocat, et du fondé de pouvoir, est extrêmement utile. 
Bentham semble avoir eu en tête ces difficultés. C'est pourquoi son œuvre se révèle si riche ${ }^{6}$. Soulignant l'ambiguïté constitutive de la notion de représentation - pouvant, dans les cas extrêmes, servir à légitimer le règne d'un despote -, il refuse de la concevoir comme une simple fiction du droit, justifiant la confiscation du pouvoir souverain. Tout son effort consiste ainsi à trouver une mécanique permettant, par l'entremise d'institutions représentatives, de donner un contenu réel au pouvoir du peuple, exercé pour le peuple et par le peuple. En ce sens, il s'agit bien pour lui de maintenir la multitude au cœur du fonctionnement des institutions. L'enjeu est donc de penser une traduction institutionnelle du pouvoir du peuple comme pouvoir souverain, qui rende en outre impossible le sacrifice de l'intérêt du grand nombre (the subjected many) par le petit nombre des dirigeants (the ruling few). C'est à la saisie de cet effort que s'attache le présent article. Aussi nous faudra-t-il comprendre comment Bentham parvient à faire du peuple souverain l'un des pouvoirs constitutionnels, faisant de ce "pouvoir constitutif » un pouvoir véritablement actuel - l'actualité, qui s'oppose ici à la virtualité, renvoyant très clairement à une forme de pouvoir du peuple en acte.

Pour cela, il nous faudra d'abord préciser la méthode particulière que Bentham met en ouvre dans sa critique des institutions politiques de son temps afin, dans un second temps, de voir comment cette critique atteint le concept de représentation. C'est une fois en possession des enjeux de cette critique, et des principes nécessaires à l'établissement d'une représentation véritable, que nous pourrons éclairer la manière dont il entend fonder une représentation politique authentique, c'est-à-dire reposant sur un exercice actuel du pouvoir politique par les membres de la communauté.

\section{I. « La saison des fictions est dorénavant terminée ${ }^{7}$}

Comprendre le sens de la critique de la représentation politique de Bentham suppose de s'arrêter sur son entreprise de réforme du droit. Il nous faut donc commencer par en dégager les fondements.

La philosophie de Bentham s'enracine dans ce qui constitue pour lui une découverte majeure : le principe d'utilité. Ce principe, selon lequel « c'est le plus grand bonheur du plus grand nombre qui doit être la mesure du juste et

\footnotetext{
6. Pour une vue d'ensemble de la pensée constitutionnelle de Bentham, voir particulièrement l'ouvrage d'E. de Champs, La Déontologie politique: Ou la pensée constitutionnelle de Jeremy Bentham, Genève, Librairie Droz, 2008. - Sur l'originalité du modèle de démocratie représentative proposé par Bentham, en tant qu'il se distingue de la démocratie libérale traditionnelle, voir notamment l'article de F. Peonidis, «Jeremy Bentham's 'unusually liberal' representative democracy », History of European Ideas, décembre 2011, vol. 37, n 4, p. 446-453.

7. Bentham, Fragment sur le gouvernement suivi de Manuel des sophismes politiques, traduction J.-P. Cléro, Paris, Bruylant-L.G.D.J., 1996, p. 124.
} 
l'injuste $»^{8}$, est identifié par Bentham comme l'axiome fondamental de la réforme qu'il entend mener. S'enracinant dans un postulat psychologique qu'il formule dès les premières pages de l'Introduction aux principes de morale et de législation", ce principe, qui «approuve ou désapprouve toute action, quelle qu'elle soit, selon la tendance qu'elle semble avoir à augmenter ou diminuer le bonheur de la partie dont l'intérêt est en jeu ou, en d'autres termes, à promouvoir ce bonheur ou à s'y opposer ${ }^{10} »$, ne s'applique toutefois pas qu'au niveau éthique. En effet, l'utilité est aussi le critère d'évaluation des actions politiques. Aussi toute mesure de gouvernement doit-elle être évaluée depuis ses effets sur le bonheur universel. Comme il le souligne notamment dans le Fragment sur le Gouvernement :

Par utilité, nous pouvons désigner un principe qui, comme tel, peut servir à présider au classement qui doit être fait des diverses institutions ou combinaisons d'institutions qui composent la matière de cette science [la théorie du droit]; comme à son contrôle ${ }^{11}$.

Le principe d'utilité est donc d'emblée descriptif et normatif. Autrement dit, si les institutions doivent être jugées à l'aune de leur capacité à maximiser le bonheur du plus grand nombre, elles doivent avoir ce même bonheur pour objet principal de leur activité.

C'est donc la perspective de l'utilité qui conduit Bentham à formuler sa critique des fictions juridiques, qui servent le plus souvent de fondement théorique au droit. Car ces entités sues fausses mais tenues pour vraies constituent selon lui autant d'obstacles à la discussion des lois sur la base de leur utilité. Deux traditions juridiques sont particulièrement visées par cette critique : d'une part, le contrat social et l'école du droit naturel, d'autre part, la Common Law, telle particulièrement que la justifie Blackstone dans son Commentaire sur les lois d'Angleterre.

La critique du contrat social, qui est à la fois la plus virulente et la plus développée par Bentham, s'enracine dans une lecture de Hume ${ }^{12}$. Comme Bentham le souligne dans une note du Fragment sur le gouvernement:

\section{Ibid., p. 87.}

9. L'ouvrage commence ainsi : «La nature a placé l'humanité sous le gouvernement de deux maitres souverains, la douleur et le plaisir. C'est à eux seuls d'indiquer ce que nous devons faire aussi bien que de déterminer ce que nous ferons. [...] Le principe d'utilité reconnaît cette sujétion et la tient pour le fondement du système dont l'objet est d'ériger l'édifice de la félicité au moyen de la raison et de la loi », Bentham, Introduction aux principes de morale et de législation, traduction par le Centre Bentham, Paris, Vrin, 2011, p. 25.

10. Ibid., p. 26.

11. Bentham Fragment sur le gouvernement, op. cit., p. 104.

12. Les réflexions de Hume sur la notion de contrat social se rencontrent particulièrement dans la seconde partie du livre III du Traité de la nature humaine, ainsi que dans son «Essai sur le contrat originel », in Essais moraux, politiques, littéraires. Et Bentham fonde sa critique du contrat social sur les mêmes arguments, bien que ceux-ci le conduisent, contrairement à Hume, à l'adoption d'une position radicale. 
Pour ma part, je me rappelle bien que, dès que j' ai lu la partie de l'ouvrage qui touche à ce sujet, je sentis des écailles me tomber des yeux [...] Je tirais ma révérence au contrat originel et le laissais à ceux qui pouvaient s'imaginer en avoir besoin pour qu'ils se distraient avec ce hochet ${ }^{3}$.

Son rejet de la fiction du contrat est entier. Devant fonder l'obéissance légitime à un gouvernement, elle ne sert en vérité qu'à produire une soumission illégitime. En effet, ce n'est pas la promesse, impliquée par l'idée de contrat, qui produit l'obéissance - autrement, les contrats ne seraient jamais rompus. La force de l'obligation est à chercher ailleurs. Si les promesses sont tenues, c'est en vertu de l'utilité que l'on trouve à les honorer. Le même raisonnement explique l'obéissance politique. Par conséquent, la promesse seule ne peut rendre raison de la permanence des gouvernements dans le temps, puisque celle-ci n'engage que l'individu qui la fait - et non ceux qui le suivent. Si l'obéissance se maintient d'une génération à l'autre, c'est que les individus trouvent à sa permanence une certaine utilité. C'est pourquoi énoncer que les gouvernements naissent de contrats originels est pour Bentham un non-sens, qui occulte le rôle réel joué par le principe d'utilité.

Quant à l'idée de droits naturels et imprescriptibles, tels que les consacre notamment la Déclaration des droits de l'homme et du citoyen de 1789 , ils n'ont d'existence que «figurative ${ }^{14}$. Comme le souligne Bentham, dire que la Nature - qui est une entité fictive - a donné à chacun un droit sur toute chose équivaut à énoncer qu'elle n'a rien donné à personne ${ }^{15}$. Autrement dit, ce droit donné par la Nature avant l'existence des lois est une «universalité de nom et non entité réelle de droit». Surtout, cette antériorité des droits naturels à toute codification juridique - puisqu'il s'agit de droits non-écrits - implique pour Bentham une absence manifeste de contenu. Les droits naturels sont, en d'autres termes, des droits vides. Ils sont donc contradictoires : si les individus ne peuvent les connaitre, les lois que l'on fait reposer sur eux ne peuvent par conséquent pas régler leurs comportements. Affirmer l'existence de droits naturels équivaut donc pour Bentham à affirmer la nullité du droit.

Enfin, en tant qu'elles confèrent aux lois un fondement sacré, devant lequel tout usage de la raison doit céder, Bentham voit dans ces fictions droit naturel et contrat social ainsi que continuité de la constitution britannique postulée par la common law - autant de moyens de refuser la

13. Fragment sur le gouvernement, op. cit., p. 123-124.

14. Voir Bentham, L'Absurdité sur des échasses, in Bentham contre les droits de l'homme, B. Binoche et J.-P. Cléro, Paris, Presses universitaires de France, 2007, p. 31.

15. Ainsi Bentham énonce-t-il : «la Nature a donné à chaque homme un droit sur chaque chose ; soit ; de là la nécessité du gouvernement et des lois humaines pour donner à chacun son propre droit : sans eux, aucun droit, quel qu'il soit, n'aurait la moindre consistance », ibid., p. 38 . 
discussion du droit sur la base de son utilité. De là la véhémence des critiques qu'il leur adresse :

Une déclaration des droits fondamentaux est à la fois obligatoirement conservatrice et anarchiste. D'un côté, elle enchaîne les générations futures à des formules sacrées et figées et elle interdit la réflexion sur leur utilité effective. D'un autre côté, elle expose toute loi à la contestation et à la désobéissance au nom de ces droits intouchables ${ }^{16}$.

En liant les mains des législateurs, ces fictions empêchent de modifier les lois sur la base de leur utilité. En instituant en outre la référence à des droits de nature, elles fondent un droit permanent de résistance au gouvernement, empêchant tout ordre politique stable. Or, dans la mesure où l'une des fins essentielles du gouvernement est d'assurer la sécurité de ses membres ${ }^{17}$, une déclaration fondamentale de droit instituant la résistance en principe conduit nécessairement à «l'anarchie». C'est pourquoi Bentham parle des droits de l'homme comme d'une boîte de Pandore.

Surtout, dans la mesure où ces fictions permettent au petit nombre des dirigeants de faire passer la poursuite de leurs intérêts pour celle de l'intérêt commun, elles servent l'usurpation du pouvoir souverain. Elles sont autant de fictions fallacieuses, des « fausseté[s] volontaire[s] qui [ont] pour objet de confisquer le pouvoir législatif par et au profit de ceux qui ne pourraient pas ou n'oseraient pas le revendiquer ouvertement, et qui ne pourraient pas l'exercer, sinon par le subterfuge qu'elle[s] [produisent] ainsi ${ }^{18} »$. Autrement dit, les gouvernants trouvent dans « la puissance fallacieuse du langage » un voile pour leurs intérêts déviants ${ }^{19}$ (sinister interests). Ce ne sont donc pas les fictions en général qu'entend condamner Bentham, mais leur usage fallacieux. C'est ainsi à partir de sa critique des fictions juridiques qu'il élabore sa théorie générale des «sophismes politiques », ces artifices de la langue qui permettent aux dirigeants de masquer leur usage illégitime du pouvoir politique.

Pourtant, dans la mesure où ces fictions sont nécessaires à la théorie du droit, Bentham ne peut se limiter à la critique de leurs mésusages. Il lui faut au contraire refonder le langage du droit, de sorte qu'il soit débarrassé de ces

16. Bentham, Fragment sur le gouvernement, op. cit., p. 32.

17. Bentham fait en effet de la sécurité l'un des objets principaux, sinon le plus important, du gouvernement. Il l'inclut d'ailleurs parfois dans la définition du principe du plus grand bonheur du plus grand nombre. Voir par exemple Introduction aux principes de morale et de législation, op. cit., p. 51 : «On a montré que le bonheur des individus, dont se compose une communauté, c'est-à-dire leurs plaisirs et leur sécurité, est la fin, et même la seule fin, que le législateur doit viser ».

18. Bentham, Fragment sur le gouvernement, op. cit., p. 172.

19. Dans Rationale of Judicial Evidence, IX, 3, 1, Bentham définit les intérêts déviants de la manière suivante : "On peut appeler "intérêts déviants" (sinister interest) ces intérêts dont l'action comme les effets poussent leur possesseur à la malhonnêteté », Works, op. cit, vol. VII, p. 385. 
sophismes. La réforme politique passe donc nécessairement par la maitrise de la langue ${ }^{20}$. Car la possibilité d'user du langage pour tromper n'est pas propre au droit, mais repose sur les propriétés du langage. Pour son fonctionnement, souligne Bentham, celui-ci a besoin de créer des entités abstraites, et de faire comme s'il s'agissait d'entités réelles. Cette création, ajoute-t-il, est «un procédé sans lequel le langage [...] ne pourrait pas $\operatorname{exister}^{21} »$. Il y a donc un usage «normal » des fictions langagières, que Bentham définit de la manière suivante :

Par ce terme, on entend désigner l'une de ces sortes d'objets dont, en toute langue, pour les buts du discours, on doit parler comme s'ils existaient ; dont on doit parler de la même façon que l'on parle des objets qui possèdent réellement l'existence [...] mais sans avoir le dessein de produire la persuasion que ces entités possèdent, chacune pour elle-même, une existence séparée, ou, pour parler précisément, une existence réelle ${ }^{22}$.

Comme telles, donc, les entités fictives ne doivent pas être condamnées. Participant de la construction du langage, elles sont en outre absolument nécessaires à l'expression d'idées complexes, c'est-à-dire d'idées ne se rapportant pas directement à des sensations physiques de peine ou de plaisir.

Mais pour être conservées, ces fictions doivent posséder un fondement réel - et non fictionnel. Reprenant ici la méthode proposée par Helvétius dans le chapitre «De l'abus des mots » de De l'esprit ${ }^{23}$, Bentham souligne que, pour avoir du sens, les entités fictives doivent pouvoir être rapportées à des «entités réelles », c'est-à-dire à des entités dont nous pouvons faire l'expérience sensible. Il faut ainsi «mettre en évidence et placer sous le regard du lecteur ces entités afin que toute personne qui voudra prendre la peine de les lire puisse attacher et annexer des idées aussi claires, correctes et complètes que possible à chacun de leurs noms ${ }^{24} \gg$. La question du rapport entre les mots et le réel dont ils doivent rendre compte est donc décisive. Par conséquent, pour qu'une entité fictive soit acceptable - c'est-à-dire pour s'assurer que l'on n'en fera pas un usage fallacieux -, il faut qu'elle entretienne « une certaine relation avec une certaine entité réelle ${ }^{25}$, relation qui puisse en outre être perçue par tout individu.

\footnotetext{
20. Sur la question des fictions chez Bentham, et plus particulière sur l'importance qu'il leur accorde dans la perspective d'une réforme du droit, voir C. Laval, Jeremy Bentham : le pouvoir des fictions, Paris, Presses universitaires de France, 1994.
}

21. De l'ontologie, et autres textes sur les fictions, J.-P. Cléro, C. Laval, P. Schofield, Paris, Seuil, 1997, p. 85.

22. Ibid., p. 87.

23. Helvétius souligne en effet dans ce chapitre la nécessité de remonter à la sensation qui a originellement donné naissance à l'idée et donc au mot, au risque sinon d'être trompé par les puissants.

24. Bentham, De l'ontologie, op. cit., p. 79.

25. Ibid., p. 165. 
Appliquée au droit, cette théorie des fictions implique que tout énoncé juridique puisse être traduit en termes de plaisir et de peine. En effet, si le plaisir et la peine sont les souverains maîtres des individus, et si l'utilité d'une action quelconque repose sur sa tendance à promouvoir ou diminuer ces sensations, il est essentiel de traduire les lois dans le langage de l'utilité. Aussi faut-il, pour réduire l'abstraction inévitable de la loi, remonter à son origine, et faire apparaître les rapports qu'elle entretient avec les corps et la répartition des peines et des plaisirs. C'est pourquoi le travail du législateur est d'abord de «neutraliser » le droit. Comme Bentham le souligne :

Ces dénominations fantastiques constituent une sorte de papier-monnaie : si nous savons à tout instant comment les échanger et comment obtenir à leur place de la monnaie de bon aloi, tout est pour le mieux. Si nous ne le pouvons pas, nous sommes trompés, et au lieu d'être les maîtres d'une connaissance réelle dont nous entendions nous pourvoir par leur moyen, nous n'avons alors que sophistique et non-sens ${ }^{26}$.

La métaphore du change utilisée par Bentham dans ce passage met bien en évidence le fait que fonder les fictions du droit passe par un travail de conversion de ces fictions en entités réelles. Mais cette idée d'une conversion s'éclaire davantage si l'on prend un exemple. Soit l'obéissance politique, que Bentham travaille dans le Fragment sur le gouvernement. Si, comme nous l'avons vu, il est impossible de comprendre l'obéissance politique en la rapportant à un contrat originel, c'est que cette fiction ne permet pas de rendre compte du phénomène d'obéissance. Pour donner un fondement réel à l'obéissance, il faut la rapporter à des actes déterminés, exprimant l'obéissance des individus à la loi. Ainsi, si l'obéissance politique caractérise «tout acte effectué conformément à l'expression de la volonté d'une personne qui gouverne $»^{27}$, le degré d'obéissance habituelle correspond quant à lui au rapport entre le nombre d'actes d'obéissance et le nombre d'actes de désobéissance. Aussi comprend-on comment une fiction du droit peut être «convertie» en actes constatables, c'est-à-dire fondée empiriquement.

On le voit, la réussite de la réforme du droit repose sur la maîtrise du langage. Et elle vise principalement deux buts, puisqu'elle doit garantir, d'une part, la possibilité pour chacun de déterminer rationnellement les conséquences d'une obligation légale et, d'autre part, l'impossibilité d'usurper le pouvoir souverain.

26. Bentham, Of Laws in General, éd. H.L.A. Hart, London, University of London, Athlone Press, 1970, p. 251-252.

27. Bentham, Fragment sur le gouvernement, op. cit., p. 115. 


\section{II. « Dans le langage des menteurs, le gouvernement est un corps représentatif $»^{28}$}

C'est encore cette réforme du droit qui conduit Bentham à condamner une certaine idée de la représentation politique. Celle qui, ne possédant aucun fondement empirique, n'est qu'un sophisme. C'est que le terme même de «représentation », souligne Bentham dès $1795^{29}$, souffre d'une indétermination constitutive :

Pour caractériser les délégués, la langue française a, comme la langue anglaise, deux mots, les représentants et les députés; l'un est susceptible d'être mal interprété, au point d'exprimer un sens directement opposé à celui qui semble avoir été celui qu'on a voulu lui donner; l'autre pas. L'un est teinté de fiction tout comme d'ambiguïté ; l'autre n'exprime rien que la simple vérite ${ }^{30}$.

Dans ce passage, il ne faut pas s'y tromper, le terme de «fiction» signifie fiction fallacieuse. La critique de la représentation repose ainsi sur la critique des fictions. Poursuivant sa critique de la représentation dans le Manuel des sophismes politiques, Bentham y souligne que l'ambiguïté de ce terme tient d'abord au fait qu'il ne renvoie à aucun acte déterminé. Car «n'importe quelle personne ou n'importe quelle chose peut être représentée par une autre. Un homme est représenté par une toile et des couleurs; l'ensemble du peuple l'est par le roi ; le roi et par conséquent le peuple le sont par un ambassadeur ${ }^{31}$. La notion de représentation renvoie donc à des réalités extrêmement variées. Or si « représenter» se dit en plusieurs sens, le sens mobilisé n'est jamais précisé. Dans les usages qui, de la fin du XVIII ${ }^{\mathrm{e}}$ siècle aux années 1820 , sont faits de l'idée de représentation politique, ni la fonction qu'occupe le représentant, ni la manière dont cette activité permet effectivement de représenter les électeurs ne sont spécifiées. Les traits définitionnels majeurs du mécanisme représentatif sont ainsi laissés indéterminés. Et l'indétermination conduit inévitablement à l'usurpation.

Par conséquent, le concept de représentation politique mobilisé par les contemporains de Bentham se range parmi les sophismes dits de «confusion». Ceux-ci reposent sur un mode de raisonnement «aérien», et permettent à ceux qui les utilisent de ne jamais nommer les réalités véritablement engagées. Leur pouvoir fallacieux se loge dans leur généralité, qui plonge intentionnellement le récepteur du discours dans un état de

28. Bentham, Garanties contre l'abus de pouvoir et autres écrits sur la liberté politique, traduction M.-L. Leroy, Paris, Presses de l'École normale supérieure, 2001, p. 89.

29. Voir par exemple L'Absurdité sur des échasses, op. cit., p. 53-54.

30. Ibid.

31. Bentham, Manuel des sophismes politiques, op. cit., p. 205. 
confusion. Et cette confusion a des effets politiques. En effet, maintenant le grand nombre dans l'ignorance, ce «charabia » de la représentation permet aux gouvernants d'user du pouvoir souverain sans s'en justifier. C'est pourquoi, du point de vue de la légitimité, lesdits représentants du peuple sont pour Bentham plus condamnables que les despotes. Il le souligne notamment dans le Manuel:

Pour être despote, l'Empereur du Maroc n'est pas imposteur; ce qu'est un parlementaire. L'empereur ne prétend pas être mandataire, agent, député, délégué, représentant; le mensonge ne fait pas partie du cortège de sa tyrannie et de son insolence; le parlementaire a toutes ces prétentions et, s'il est propriétaire dans un bourg, il ment. Oui, c'est un mandataire, mais par parjure. Oui, c'est un agent ; mais pour son compte. Un représentant du peuple, oui ; mais comme Mr Kemble représente Macbeth. Un député certes, parce qu'il n'a pas été au pouvoir du peuple de députer ou de déléguer quelqu'un d'autre. Député ? Délégué ? Il ne revendique aucun de ces titres, sinon par rhétorique et quand il ne peut pas faire autrement. «Députation» renvoie à une question de fait et met l'accent sur le petit nombre des électeurs et leur manque de liberté. «Représentation» est un mot plus commode. Il tient à l'écart les actes des électeurs ${ }^{32}$.

Ainsi, contrairement au parlementaire, qui prétend être le mandataire du peuple souverain et exercer le pouvoir en son nom, le despote n'a pas besoin de recourir aux sophismes pour justifier son pouvoir. En d'autres termes, l'usage de la fiction de «représentation» doit rendre invisible la confiscation du pouvoir opérée par le petit nombre des dirigeants. Ce faisant, il justifie la mise à l'écart des " actes des électeurs », rendant impossible toute traduction empirique de leur représentation. C'est pourquoi, comme l'affirme Bentham dans sa $4^{\mathrm{è}}$ «Lettre sur la liberté de la discussion publique dans les réunions libres », le gouvernement représentatif n'a de représentatif que le nom ${ }^{33}$.

Et ce sophisme de la représentation se révèle d'autant plus prégnant qu'il s'ancre dans la théorie de la représentation virtuelle (virtual representation), justifiant principiellement l'exclusion du plus grand nombre. La critique de la fiction de représentation vise donc plus directement l'idée de représentation virtuelle. Celle-ci se forge dans le sillage de la Révolution américaine. Lorsqu'en 1765, le Parlement britannique décide d'imposer aux colonies américaines une nouvelle taxe (conséquence du Stamp Act), cette décision provoque des vives réactions de protestation. En effet, adoptée par un parlement où ne siège aucun représentant élu par les colonies, cette nouvelle taxe est perçue par l'opinion américaine comme profondément injuste. C'est dans ce contexte qu'est reprise la formule «no taxation without representation», qui deviendra le

32. Ibid., p. 205.

33. Voir Bentham, Garanties contre les abus de pouvoir, op. cit., p. 89. 
mot d'ordre des insurgés. Son sens est clair: personne n'est représenté sans élire son représentant. C'est en réponse à ces protestations qu'émerge la doctrine de la représentation virtuelle - théorisée notamment par S. Jenyns et $\mathrm{T}$. Whateley. Les partisans de cette doctrine concèdent aux colons américains qu'il est vrai qu'en un certain sens, ils ne sont pas représentés. N'élisant pas de députés au Parlement britannique, les colonies américaines ne sont en effet pas actuellement représentées. Mais ils soulignent dans le même temps que cette représentation actuelle n'est pas essentielle. Car ce qui compte n'est pas d'être représenté actuellement, mais de l'être virtuellement. Et les colonies américaines le sont, puisque leur intérêt est inclus dans celui de la communauté. Cette idée d'une représentation virtuelle se fonde ainsi sur le principe d'un intérêt commun transcendant les intérêts particuliers des membres de la communauté. À cet égard, la tâche de l'assemblée des représentants du peuple est précisément de faire émerger cet intérêt. C'est pourquoi les tenants de cette version de la représentation peuvent affirmer qu'il n'est pas nécessaire que toutes les parties de population envoient des représentants pour être représentées. Cette théorie permet, en outre, de justifier l'exclusion du suffrage d'une large part de la population britannique - la situation des colonies étant comparée à celle des villes de Birmingham et de Manchester qui, dans la seconde moitié du XVIII ${ }^{\mathrm{e}}$ siècle, n'envoyaient pas non plus de représentant à la chambre des Communes.

La représentation virtuelle trouve probablement son expression la plus fameuse sous le plume de Burke, qui souligne, dans son «Discours aux électeurs de Bristol » :

Le Parlement n'est pas un congrès d'ambassadeurs envoyés par des intérêts différents et hostiles, intérêts que chacun doit défendre, en tant que mandataire et avocat, contre d'autres mandataires et d'autres avocats ; le Parlement est l'assemblée délibérative d'une seule nation, mue par un seul intérêt, celui du tout ; et où ce ne sont ni les objectifs locaux, ni les préjugés locaux qui doivent servir de guide mais le bien général, produit de la raison générale du tout ${ }^{34}$.

On le voit, dans la représentation virtuelle, si le représentant doit agir pour le peuple, il n'est en aucun cas attendu de lui qu'il agisse comme le peuple. Aussi peut-il, s'il le juge nécessaire, aller à l'encontre des vœux clairement exprimés de ses électeurs. Le travail du représentant est donc, au sein de l'assemblée, de délibérer sur l'intérêt général, ce qui ne saurait être possible s'il était lié par les opinions des individus qu'il représente. Leur opinion n'a ainsi aucun rôle à jouer dans le processus délibératif : une fois élu, le représentant est libre de ses décisions.

34. Burke, "Speech to the electors of Bristol», in Burke's Politics. Selected Writings and Speeches, R.J.S Hoffmann et P. Levack (éd.), New York, A.A. Knopf, 1949, p. 115. 
Comprendre la critique de la représentation politique formulée par Bentham suppose ainsi de l'inscrire dans le contexte de cette opposition entre représentation virtuelle et représentation actuelle. Car le choix de Bentham de parler de représentation actuelle est d'abord une manière de réinvestir l'un des pôles de ce débat. Cet arrière-plan est particulièrement lisible dans le Manuel des sophismes politiques, où Bentham affirme, non sans ironie :

Si tous ceux qui se contentent si bien d'une représentation virtuelle et sans réalité pouvaient aussi bien se satisfaire d'une perception virtuelle des impôts, d'une part, et d'une contribution virtuelle d'autre part, tout irait bien. Mais ce n'est malheureusement pas le cas. Le paiement des impôts n'est que trop réel, tandis que le fondement par lequel on prétend même justifier leurs exactions, la représentation, est d'une fausseté si incontestablement établie et de notoriété si générale qu'en affirmer l'existence est d'une ironie féroce ${ }^{35}$.

S'inscrivant résolument dans le sillage du mot d'ordre "no taxation without representation », Bentham souligne ici combien le subterfuge de la représentation est insultant à l'égard du peuple, puisque les arguments en faveur de la représentation virtuelle laissent transparaître l'hypocrisie qui les fonde. La représentation politique, au moins lorsqu'elle est associée à l'idée de virtualité, est donc bien un sophisme politique. Il conduit à faire de l'usurpation du pouvoir la norme. À cet égard, la force du sophisme de la représentation virtuelle est qu'il peut servir à justifier n'importe quel type de régime politique :

C'est d'une de ces anomalies qu'est née la doctrine de la représentation virtuelle : phrase avec laquelle on pourrait prouver la constitution marocaine aussi libre que l'anglaise. En Angleterre c'est une partie du peuple qui représente l'autre : et au Maroc c'est l'Empereur qui représente le peuple ${ }^{36}$.

Si la notion de représentation est une fiction nuisible, c'est donc bien qu'elle ne possède aucun fondement réel. Aussi l'enjeu est-il, une fois le sophisme démasqué, de déterminer comment circonscrire son usage légitime. Autrement dit, il importe dorénavant de trouver ces entités réelles, les «actes des électeurs » auxquels renvoie la «députation» que Bentham entend substituer à la représentation.

35. Bentham, Manuel des sophismes politiques, op. cit., p. 303.

36. Bentham, «Considérations d'un Anglais sur la composition des États Généraux », in Rights, Representation and Reform, The Collected Works of Jeremy Bentham, éd. P. Schofield, C. Pease-Watkin et C. Blamires, Oxford, Oxford University Press, 2002, p. 86 . 


\section{III. «Ce n'est que dans la mesure où le peuple choisit actuellement ses représentants [...] que de tels représentants peuvent être utiles ${ }^{37}$}

Quelle forme prend donc la représentation actuelle? Pour le comprendre, il est nécessaire de mettre en évidence la manière dont la fiction de représentation peut être rapportée à des entités réelles. Il nous faut donc trouver ces actes réels dans lesquels s'ancre la députation.

Pour cela, le plus aisé est de se rapporter au modèle de l'obéissance. En effet, de même que l'obéissance doit renvoyer aux actes réels d'obéissance des sujets, la députation doit quant à elle renvoyer aux actes par lesquels les individus manifestent explicitement leur adhésion à leur représentant. Ces actes sont de deux sortes: d'une part, l'expression des électeurs par le suffrage; d'autre part, la manifestation non institutionnelle des intérêts au sein de l'opinion publique. On notera à cet égard que l'existence d'une représentation non sophistique a pour condition l'établissement du suffrage universel, ainsi que la garantie de la liberté de la presse et d'expression. Bentham insiste en de nombreux endroits ${ }^{38}$ sur le fait que le droit de vote n'a de sens qu'à condition qu'existe une presse libre. Comme il le souligne notamment dans sa seconde «Lettre sur la liberté de la discussion publique dans les réunions libres », le droit de vote ne peut produire aucun bien si les électeurs sont privés de " [1'] occasion d'apprendre à s'exercer mentalement et à acquérir cet instrument d'indépendance qui seul permet de distinguer l'homme d'une marionnette ${ }^{39} \gg$. Conditions nécessaires à l'existence d'une députation, le droit de vote et la liberté de la presse doivent donc permettre la manifestation réelle, et clairement lisible, de l'adhésion des individus aux décisions du pouvoir souverain.

Mais il nous faut encore préciser sur quoi porte précisément cette adhésion. Autrement dit, il nous faut comprendre ce qui, dans la représentation actuelle, doit être représenté. Or dans la mesure où le principe d'utilité implique la prise en compte de tous les intérêts, l'actualité de la représentation n'a d'autre objet que la coïncidence entre les intérêts réels des individus et leur représentation au sein de l'assemblée. Elle doit donc être comprise en termes de figuration des intérêts. Cette exigence pose des difficultés certaines. Car dire qu'il faut une représentation actuelle des intérêts implique qu'il n'existe pas, contrairement à ce que postule la théorie de la représentation virtuelle, un intérêt général pouvant être formulé indépendamment de l'expression des intérêts particuliers. On peut dès lors se demander si la formulation de l'intérêt commun est réductible à la somme des intérêts particuliers exprimés - cette première question impliquant de

37. Bentham, Manuel des sophismes politiques, op. cit., p. 303.

38. Voir par exemple «Division of Power», in Right, Representation and Reform, op. cit., p. 409.

39. Bentham, Garanties contre les abus de pouvoir, op. cit., p. 76. 
déterminer la manière de figurer dans leur diversité réelle tous les intérêts d'une communauté donnée.

En premier lieu, il apparaît clairement que l'intérêt général ne saurait être conçu indépendamment des intérêts formulés par les membres d'une communauté. Comme le souligne Bentham dès l'Introduction aux principes de morale et de législation : "Qu'est-ce que l'intérêt de la communauté ? La somme des intérêts des divers membres qui la composent ${ }^{40}$. Cet intérêt trouve donc son expression adéquate dans la somme de leurs suffrages. En ce sens, l'intérêt commun est produit mécaniquement par le vote. En effet, dans le cadre d'un vote à scrutin secret, à l'occasion duquel chaque individu peut librement choisir le candidat qu'il estime le plus à même de porter son intérêt, le nombre de voix obtenu par un candidat exprime le degré de coïncidence entre sa conception des intérêts du plus grand nombre et celle du plus grand nombre lui-même. Pourtant, dans cette perspective, c'est bien la somme des intérêts particuliers qui semble conduire à l'élection d'un député, et donc, par extension, à la détermination de l'intérêt commun.

Deux éléments supplémentaires compliquent néanmoins ce que Bentham comprend comme étant l'intérêt commun. D'une part, il souligne qu'il y a toujours des questions politiques pour lesquelles un individu donné n'a pas d'intérêt en jeu. Dans ce cas, nous dit-il, son choix sera guidé par « un intérêt secondaire », qui l'amènera à considérer ce qui, faute d'avoir un intérêt pour lui, lui semble pourtant le meilleur pour la société dans son ensemble. Aussi Bentham souligne-t-il :

Il s'en faut de beaucoup que chacun se trouvât personnellement intéressé dans toute opération de gouvernement qui se trouverait dans le cas d'être proposée. Mais après son intérêt personnel, et ceux de la petite sphère de ses liaisons particulières, viendrait même pour les plus égoïstes, l'intérêt général de la société. [...] Ainsi cette formule, ce qui lui serait le plus avantageux, savoir à chaque individu, servirait à représenter inclusivement à cet égard l'idée de ce qui selon lui devrait paraître le plus avantageux à la sociétét ${ }^{41}$.

Par son vote, donc, l'électeur n'exprime pas seulement son intérêt particulier, mais partage dans le même temps une certaine conception du bien commun. D'autre part, même dans le cas d'un individu mû uniquement par des intérêts égoïstes, le mécanisme électif l'oblige à accorder son suffrage à ce qui lui apparaît comme étant la conception la plus large possible de l'intérêt. En effet, dans la mesure où il ne peut y avoir coïncidence parfaite entre l'intérêt d'un individu et ceux portés par un candidat, l'électeur choisira rationnellement de voter pour celui qui paraît posséder la conception de l'intérêt général la plus large. De la sorte, celui-ci maximise ses chances de voir son propre bonheur réalisé. Par son vote,

40. Bentham, IPML, op. cit., p. 27.

41. Bentham, «Considérations d'un Anglois sur la composition des États Généraux», in Right, Representation and Reform, op. cit., p. 71. 
chaque individu ne promeut donc pas seulement ce qui est bon pour lui, mais aussi la part de son intérêt particulier qui recoupe l'intérêt général. Aussi faut-il distinguer les motifs du vote, qui relèvent bien de l'intérêt particulier, et l'intérêt exprimé par le vote, qui n'est autre que la perception, par l'individu, de ce qu'est l'intérêt du plus grand nombre ${ }^{42}$. Bentham attend donc du processus électoral qu'il produise mécaniquement la promotion de l'intérêt universel ${ }^{43}$.

Il apparaît ainsi que l'intérêt général n'est autre, pour Bentham, que la conception majoritaire de ce même intérêt, mise en évidence par le scrutin. C'est pourquoi sa formulation n'est pas réductible à la somme des intérêts particuliers. Bentham souligne en outre que, puisque cette majorité est constituée du peuple, c'est-à-dire des individus qui ont le plus intérêt à ce que l'intérêt général l'emporte, l'intérêt qu'elle exprime est nécessairement juste. C'est donc à la majorité qu'il revient d'exprimer l'intérêt de la communauté.

On comprend ainsi en quel sens la représentation doit être actuelle : si l'intérêt général n'est découvert que par l'élection, son contenu ne peut pas préexister à sa formulation dans le scrutin. Cette expression de l'intérêt général par les électeurs doit, en outre, conduire à une figuration fidèle au sein de l'assemblée. Ainsi Bentham souligne-t-il : "La composition de l'assemblée législative sera meilleure à proportion du nombre de points de contact qu'elle aura avec la nation; autrement dit, en proportion de la similitude entre les intérêts qu'elle manifeste et ceux de la communauté ${ }^{44}$. On le voit, la représentation, pour être actuelle, doit manifester le plus fidèlement possible l'intérêt général tel qu'il s'exprime, à un moment donné, au sein de la communauté.

C'est aussi pour garantir cette fidélité de la représentation que Bentham encadre assez strictement l'exercice des mandats. Car l'élection ne saurait être comprise comme une autorisation illimitée d'agir au nom des électeurs. La députation, qu'encadre le vote, se limite à un mandat donné à l'individu élu pour porter à l'assemblée les intérêts de ses mandants. Bentham le souligne largement lorsqu'il énonce :

Qu'est-ce qu'une élection? C'est une déclaration solennelle énonçant qu'au moment de sa formulation un certain homme jouit de la confiance de ses électeurs. Mais cette déclaration ne possède pas une vertu miraculeuse, qui garantirait le caractère de cet homme et les actions futures qu'il entreprendra ${ }^{45}$.

42. Cette distinction entre le motif du vote et l'intérêt qu'il exprime est similaire à la distinction, sur le plan moral, entre le motif d'une action, qui n'a pas d'incidence sur sa valeur morale, et ses conséquences, qui seules doivent être considérées.

43. Voir les analyses d'A. Guillot sur la mécanique du vote dans Jeremy Bentham : le peuple comme fiction, Paris, Publications de la Sorbonne, 2014, p. 201 et $s q$.

44. Bentham, Political Tactics, CW, éd. M. James, C. Blamires et C. Pease-Watkin, Oxford, Clarendon Press, 1999, p. 15.

45. Ibid., p. 16. 
On le voit, dans la mesure où rien ne garantit la manière dont l'élu agira dans le futur, l'électeur doit pouvoir, dès lors qu'il le juge nécessaire, corriger ou réaffirmer l'expression de son intérêt au sein de l'assemblée. C'est la raison pour laquelle Bentham propose l'adoption de deux mécanismes institutionnels supplémentaires. D'une part, le choix de mandats courts, non-renouvelables immédiatement ${ }^{46}$. D'autre part, la révocabilité des élus, sous certaines conditions garantissant la continuité de l'exercice du pouvoir ${ }^{47}$.

Pour être fondée, la représentation doit donc en premier lieu satisfaire une exigence de similitude entre la représentation nationale à l'assemblée et les représentés. Mais cette affirmation n'est pas suffisante. Si l'on comprend aisément ce qui fonde cette exigence de ressemblance, il est en revanche plus difficile de comprendre comment donner au lien de représentation une actualité véritable, s'ajustant à tout moment à une opinion publique mouvante.

De fait, dans la mesure où la coïncidence entre la perception de l'intérêt général exprimé par la majorité et son expression dans la représentation doit être la plus grande possible, elle ne saurait limiter sa manifestation au vote. L'exigence d'une représentation actuelle suppose ainsi qu'il soit possible, à tout moment, d'ajuster la représentation. Cette exigence se traduit, comme nous l'avons vu, par une certaine flexibilité de la députation. Mais elle conduit aussi Bentham à penser une mécanique institutionnelle devant conduire à la probité des détenteurs du pouvoir politique. En ce sens, l'arrangement institutionnel qu'il propose vise au moins deux buts : d'une part, assurer l'ajustement de la représentation à l'intérêt général au moment où il est formulé ; d'autre part, obliger les députés à agir conformément à l'intérêt général.

Dans la démocratie représentative pensée par Bentham, la méfiance à l'égard des détenteurs du pouvoir doit permettre d'atteindre ces deux buts. Celle-ci est d'ailleurs érigée en principe : «On reproche au régime de la publicité qu'il soit un système de méfiance ? Cela est vrai, et toute bonne institution politique devrait reposer sur ce principe ${ }^{48}$. La publicité des actes du Parlement est décisive : elle seule garantit la surveillance des députés par le peuple - ce qui doit conduire ces derniers à n'agir qu'en fonction de l'intérêt commun. En ce sens, la publicité répond au problème politique de l'opposition entre la fin légitime de tout gouvernement, qui n'est autre que la poursuite du «plus grand bonheur du plus grand nombre ${ }^{49}$, et la fin réellement poursuivie par les gouvernants, leur propre intérêt. Si ce soupçon à l'endroit des détenteurs du pouvoir s'enracine dans un principe

46. Sur ces questions, voir Jeremy Bentham, Constitutional Code, Vol. 1, CW, ed. F. Rosen et J.H. Burns, Oxford, Oxford University Press, 1983, p. 57 et p. 72.

47. Sur ce point, voir notamment Constitutional Code, op. cit., p. 29 et sq, et p. 117.

48. Bentham, Political Tactics, op. cit., p. 37.

49. Bentham l'exprime en de nombreux endroits; voir par exemple IPML, Constitutional Code, First Principles Prepatory to Constitutional Code. 
psychologique, en fonction duquel «dans le cœur de tout être humain, l'intérêt égoïste prend le pas sur tous les autres intérêts agrégés ${ }^{50}$, il ne s'agit pas de le condamner. Seulement, il est nécessaire de reconnaitre qu'il y a le plus souvent compétition, voire conflit, entre l'intérêt d'un individu et celui des autres membres de la communauté. C'est ainsi la reconnaissance de l'opposition naturelle entre l'intérêt des gouvernants et celui des gouvernés qui conduit Bentham à faire du soupçon un principe politique. Car en l'absence des garanties nécessaires, il est quasi certain que l'intérêt du plus grand nombre sera sacrifié.

Comment, dès lors, rendre impossible le sacrifice de l'intérêt du plus grand nombre? Pour Bentham :

La nature du cas n'admet qu'une seule méthode, qui est de détruire l'influence et l'effet des intérêts déviants (sinister interest), auxquels la situation de l'individu peut l'exposer : cela étant réalisé, il sera par conséquent virtuellement débarrassé de ces intérêts sous toutes leurs formes : et il ne restera comme seul intérêt déterminant sa conduite que son intérêt droit et convenable : cette part de son intérêt qui recoupe l'intérêt universel (ce qui revient au même que de dire qu'il ne lui restera que cet intérêt qui est conforme à l'intérêt universel tel qu'il se rencontre dans la communauté $)^{51}$.

L'enjeu est donc de contenir, chez les représentants, la tendance à sacrifier l'intérêt universel, de sorte que seule la part de leur intérêt qui recoupe l'intérêt de la communauté puisse motiver leur action. Il faut ainsi, par un mécanisme similaire à celui s'exerçant dans le vote, produire «l'identification des intérêts », cette «liaison manifeste et indissoluble» entre «l'intérêt de l'employé et son devoir » qui soit le rendra véritablement vertueux, soit le conduira à agir «tout de même que s'il l'était ${ }^{52}$ ». Bentham nomme ce processus de production de comportements vertueux «maximisation de l'aptitude morale». L'aptitude morale est, chez un individu, « une qualité négative [...] caractérisée par l'absence, autant qu'il est possible [...] de la propension au sacrifice de tous les autres intérêts au profit de ce qui est son intérêt prédominant $»^{53}$. Elle conditionne donc la possibilité que soit suivie l'utilité. Pour la maximiser, il est nécessaire « de placer [les détenteurs du pouvoir] dans une situation telle qu'alors qu'ils n'ont aucune perspective d'améliorer de quelque façon leur propre bonheur en diminuant celui du plus grand nombre, ils ont la possibilité d'augmenter leur bonheur en proportion de l'accroissement du bonheur du plus grand nombre qu'ils seront capables de réaliser $»^{54}$. C'est pour y parvenir que la

50. Bentham, First Principles Prepatory to Constitutional Code, CW, ed. P. Schofield, Oxford, Clarendon Press, 1989, p. 233.

51. Ibid., p. 236.

52. Bentham, «Considérations d'un Anglais sur la composition des États Généraux », op. cit., p. 91-92.

53. Bentham, First Principles Preparatory to Constitutional Code, CW, op. cit., p. 13.

54. Ibid., p. 14. 
publicité est déterminante. En effet, garantir l'aptitude morale des membres de la députation suppose que ceux aux dépens desquels se fait le sacrifice aient les moyens de l'exposer et de le punir. Le contrôle s'effectue ainsi de deux manières : soit en punissant directement le gouvernant mis en cause dont la culpabilité, si elle est avérée à la suite d'un jugement, doit donner lieu à une sanction politique ; soit en exerçant ce que Bentham nomme la sanction morale ou populaire.

En dépit de toute une série de dispositifs institutionnels devant encadrer l'exercice du pouvoir politique en vue de son exercice régulier ${ }^{55}$, la sanction morale apparaît à Bentham comme la contre-force la plus efficace. Celle-ci s'exerce à travers l'activité du «tribunal de l'opinion publique ». Ce tribunal est notamment décrit dans le texte Garanties contre les abus de pouvoir ${ }^{56}$. Instance non officielle d'expression du peuple, il se caractérise d'abord par son extension; en effet, celui-ci «se compose de toutes les personnes physiques capables de s'intéresser aux affaires publiques, et de considérer que celles-ci sont les dépositaires d'un pouvoir qu'elles exercent en leur propre nom et en celui de tous les autres membres de la communauté $»^{57}$. Ce tribunal est donc extrêmement inclusif, puisqu'il s'étend, au moins virtuellement, à tous les membres de la communauté - y compris les individus exclus du suffrage, qu'il s'agisse des femmes ou des étrangers ${ }^{58}$. Par ailleurs, son outil principal, et le plus efficace, est la presse - dont Bentham souligne qu'elle doit être la plus variée et la plus diffusée possible. Ainsi souligne-t-il qu'il faut considérer la presse «non seulement comme l'organe propre au tribunal de l'opinion publique, mais comme le seul de ses organes dont l'activité est toujours visible ${ }^{59} \gg$. Ce tribunal se caractérise donc aussi par sa visibilité accrue, laquelle permet, précisément, de manifester en temps réel l'état de l'opinion publique. Enfin, cet organe d'expression du peuple doit exercer la sanction morale, en raison de la rectitude de ses jugements. En effet, si Bentham souligne qu'on ne peut jamais être assuré de la rectitude parfaite de ses décisions, la probabilité fait apparaître qu'elles sont celles qui, par nature, se rapprochent le plus de

55. Bentham appelle «moyens directs» ces mécanismes devant agir directement sur les détenteurs du pouvoir politique. Ils sont au nombre de quatre : minimiser le pouvoir entre les mains de chaque fonctionnaire ; minimiser la quantité d'argent entre les mains de chaque fonctionnaire ; minimiser les salaires ; exclure le «factitious dignity », c'est-à-dire ne pas accorder d'honneurs spéciaux aux dépositaires du pouvoir public. Il leur oppose les moyens indirects, ceux possédés par la classe dirigée. Sur ce point, voir notamment First Principles Preparatory to Constitutional Code, CW, op. cit., p. 29 et sq.

56. On trouve de très nombreuses mentions de ce tribunal dans l'œuvre de Bentham. Voir par exemple Constitutional Code, "Principles of Judicial Procedure », in The Works of Jeremy Bentham, éd. John Bowring, Edimbourg, 1843, II, p. 141 et 147, « Rationale of Judicial Evidence », Works, VI, p. 357.

57. Bentham, Garanties contre les abus de pouvoir, op. cit., p. 147.

58. Bentham semble parfois envisager une extension de ce tribunal à l'ensemble des membres de l'humanité, comme en attestent par exemple ses Principles of International Law.

59. Bentham, Garanties contre les abus de pouvoir, op. cit., p. 129. 
l'intérêt universel ${ }^{60}$. Aussi le tribunal de l'opinion publique est-il «le seul corps politique dont l'intérêt n'est jamais en désaccord, mais est au contraire toujours en accord avec l'intérêt du plus grand nombre des membres de la communauté en question ${ }^{61}$. La sanction morale, relayée par l'activité du tribunal de l'opinion publique, doit donc faire qu'il soit dans l'intérêt des gouvernants de poursuivre l'intérêt du plus grand nombre. C'est donc le public qui, par sa surveillance permanente, doit produire l'identification des intérêts.

Mais l'activité du tribunal de l'opinion publique est aussi décisive pour la figuration de l'intérêt universel. Puisque son activité est constante, il est l'organe le plus à même d'exprimer fidèlement les intérêts du plus grand nombre. En ce sens, nous dit Bentham, les membres actifs de ce tribunal, c'est-à-dire ceux qui écrivent et parlent publiquement, "sont les vrais représentants, et non les représentants fantoches, de tous ceux qui achètent ou lisent avec sympathie les publications qui sont le produit de leurs travaux respectifs $\gg{ }^{62}$. Le tribunal de l'opinion publique, moyen d'organiser et de rendre effective l'influence du peuple sur la législation, constitue donc le canal principal d'expression de la volonté du peuple, qu'il faut écouter si l'on veut que les lois positives soient respectées - c'est-à-dire que ne soit pas trop affaiblie la disposition à l'obéissance.

On le voit, l'exigence de figuration d'un intérêt universel par définition mouvant, dont l'expression à l'assemblée doit sans cesse être ajustée, et le soupçon à l'égard des détenteurs du pouvoir érigé en principe, conduisent Bentham à mettre la députation sous la surveillance active des membres de la communauté. Ainsi comprend-on que le véritable détenteur du pouvoir souverain n'est autre que le peuple. Cela est particulièrement lisible dans la distribution des pouvoirs au sein de la démocratie représentative pensée par Bentham dans le Constitutional Code. En effet, distinguant deux types de pouvoir, le constitutif et l'opératoire - ce dernier étant à son tour divisé selon la tripartition classique entre pouvoir législatif, pouvoir administratif ou exécutif et pouvoir judiciaire -, Bentham fait du pouvoir constitutif le pouvoir souverain. Or ce pouvoir souverain, compris comme pouvoir du peuple, est le vrai détenteur du pouvoir. Et il doit être, pour cette raison, un pouvoir actif. D'ailleurs, Bentham souligne que si le pouvoir opératif n'était pas soumis au contrôle du pouvoir constitutif, alors «être un représentant (trustee) fidèle au peuple ou un représentant indigne serait une seule et

60. Le raisonnement est donc le même que celui qui justifiait la justesse de l'intérêt exprimé par la majorité dans le vote. En effet, dans la mesure où la connaissance est toujours de l'ordre du probable, même s'il existait un intérêt général «vrai », nous ne pourrions jamais être assurés de le connaître. C'est pourquoi il faut se contenter de la conception de cet intérêt qui a le plus de chance d'être juste, à savoir celle qu'exprime la majorité. Autrement dit, il faut accepter comme juste la perception que la majorité se fait de l'intérêt du plus grand nombre, car il s'agit du seul corps qui se compose, précisément, du plus grand nombre.

61. Bentham, Garanties contre les abus de pouvoir, op. cit., p. 159.

62. Ibid., p. 148. 
même chose ${ }^{63}$. Autrement dit, c'est bien cette activité maintenue du pouvoir constitutif parallèlement à l'exercice courant du pouvoir qui garantit la conformité de cet exercice aux prescriptions de l'utilité. Aussi apparaît-il que l'exigence d'actualité ne touche pas seulement la représentation de l'intérêt général au sein de l'assemblée. Celle-ci, bien qu'essentielle, n'est en fait qu'une des manières d'incarner l'actualité du pouvoir constitutif. C'est pourquoi chez Bentham, et contrairement à d'autres modèles de gouvernement représentatif, le pouvoir constitutif ne joue pas seulement un rôle fondateur - le peuple étant identifié comme origine d'un pouvoir délégué soit pour toujours, soit pour un laps de temps limité, pendant lequel les gouvernants sont libres d'agir comme ils l'entendent. Au contraire, chez Bentham, le peuple constitue un principe actif, et même le plus éminent de sa théorie constitutionnelle. Ainsi comprend-on qu'il soit essentiel de s'assurer qu'il possède une certaine actualité.

En outre, il nous semble que Bentham, à travers son concept de représentation actuelle, propose une réponse possible aux difficultés posées par le concept de représentation politique. Ainsi voit-on que, pour lui, une conception démocratique de la représentation implique que soit représenté l'intérêt universel, tel que l'exprime à un moment donné le plus grand nombre. Et cette représentation ne saurait être réalisée sans la plus grande liberté d'expression accordée au peuple, et la mise sous surveillance de l'activité des représentants, l'une et l'autre renforçant l'identification entre le représentant et le représenté. En ce sens, il y a bien chez Bentham une théorie de la représentation, bien que la représentation elle-même soit toujours approximative, en raison de la fixation dont elle a besoin pour être figurée, mais aussi de son expression dans au moins deux canaux différenciés - l'assemblée nationale et le tribunal de l'opinion publique dont on ne peut jamais garantir la coïncidence. 